\title{
The AstraZeneca affair. A litmus test of information disorder in the Italian hybrid media ecosystem
}

\author{
Federico Pilati (IULM University, Milan, Italy - federico.pilati@studenti.iulm.it) and Guido \\ Anselmi (University of Pavia, Pavia, Italy-guido.anselmi@ unipv.it)
}

In this paper we seek to demonstrate how a variety of information disorder phenomena comes to be at the intersection of legacy and social media interaction. To do so, we collected more than 750.000 tweets and 30.000 news articles related to the adoption of AstraZeneca vaccine in Italy for a period of six months $\left(1^{\text {st }}\right.$ January $2021-30^{\text {th }}$ June 2021). Initially, using timestamps of publications and tweets, we tracked the pace of public debate. Then, using a mixed methods approach, we investigated Twitter reaction during the climax of attention toward AstraZeneca. Acting as a litmus test, our study reveals three different but intertwined information disorder phenomena: first, the vaccine debate exhibit a flat progression with few condensed peaks of attention (acceleration phenomenon); second, the two main peaks that involve both journalistic coverage and Twitter discussion generate from news of suspect deaths related to AstraZeneca (sensationalisation phenomenon); and finally, the report of suspect deaths news by mainstream media accounts on Twitter correlates with a polarized and ideological reaction of the connected publics (fragmentation phenomenon). These results highlight how a direct implication of the hybrid media ecosystem's actual configuration could be a resistance in the formation of a public arena capable of sustaining a prolonged and effective debate, particularly with respect to controversial societal issues such as those related to the covid-19 vaccination campaign.

Keywords: Infodemic; Covid-19; AstraZeneca.

\section{Hybrid Media Ecosystems, Information Disorder, and the Covid-19 Infodemic: is there a link?}

One of the most significant aspects of the current epidemic is certainly its uniqueness with respect to the huge amount of information produced and circulated off- and on- line. The covid-19 "infodemic" (i.e., an overabundance of information, reliable or not, related to a specific topic) has indeed emerged as a central issue both for research activities and public policies efforts (Zarocostas 2020). Since its very foundation, infodemiology identified the web as the main medium related to an excessive creation and dissemination of unverified contents (Eysenbach 2002) and even during the current days this view seems to be widely present in the scientific debate (Cinelli et al. 2020). Although this perspective is essential to understand the general impact of the infodemic (Gallotti et al. 2020), an excessive focus on online disinformation does not seem to capture other aspects of the communicational crisis emerged around the covid-19 (Righetti et al. 2021). As examples the challenges of institutional communication (Lovari and Righetti 2020), the politicization and polarization of public debate (Boccia Artieri 2020; Iannelli et al. 2020) or the scientific dissemination activities of mainstream media (Saracino 2020) are as relevant as the infodemic itself.

However, if we look at the infodemic metaphor as a heuristic for navigating the current communication crisis (Camargo and Felix 2021), the huge volume of news and online conversations that have aggregated around covid-19 allows us to dig deeper into the logic of hybrid media 
ecosystems (Chadwick 2017). This way, by using covid-19 infodemic as an unprecedented source of data (Gruzd et al. 2021), we can better investigate a variety of information disorder phenomena that comes to be at the intersection of legacy and social media interaction (Wardle 2017).

A first pair of hybrid media ecosystems' information disorder - i.e., the acceleration and sensationalisation of public debate - are related to the digital attention economy (Webster 2014), which pushes newsmakers to relentlessly chase the visibility metrics on which web search engines are based (Graham 2017). Such a configuration of online flows is made possible by the new web infrastructure (Gray et al. 2020), that also accentuate the acceleration and turnover of ephemeral information bubbles (Castaldo et al. 2021): by saturating media discourse with continuous and different spikes of attention, the "junk news" regime (Venturini 2019) obstacles a prolonged and focused debate, thus having a negative repercussion on the discussion of complex topics as the ones related to covid-19.

Another information disorder phenomenon related to the interplay between legacy and social media is the fragmentation of public debate. The presence of online echo-chambers and their ideological polarization (Colleoni et al. 2014) is accentuated by the emission of rumours or incomplete news (Rojiecki and Meraz 2016) that are promptly manipulated or amplified by political factions (Marwick and Lewis 2017). As for the covid-19 debate, the pre-existing social media partisanships (Caliandro, Anselmi and Sturiale 2020) could be exacerbated by the issuance of emotional contents that trigger an affective reaction in the connected publics (Boccia Artieri et al. 2021).

\section{The AstraZeneca affair}

The vaccination campaign against covid-19 started in Italy in the last days of 2020 and expanded to the whole national population from March 2021. It was precisely early in this month that some cases of possible strong adverse reaction to the vaccine patented by AstraZeneca were firstly reported. As a result, the government decided to stop the campaign (from $16^{\text {th }}$ to $19^{\text {th }}$ March) and restarted it only after the Italian Drugs Agency certified the absence of any link between the signalled deaths and the seized AstraZeneca vaccine batches. However, a few weeks later (April $7^{\text {th }}$ ) the European Medicines Agency published a document that confirmed a weak correlation between rare thrombosis and AstraZeneca, suggesting the vaccine's use only for the part of the population older than 60 years. Following the EMA's warning, the Italian vaccination campaign resumed by prioritising the over60s but left unaffected the possibility of using the serum also for younger citizens. In June: following the participation in an open vaccination event, an 18-year-old girl died in Genoa from a rare thrombosis linked to AstraZeneca. A few days after the dramatic event, the administration of AstraZeneca vaccine, previously renamed Vaxzevria, stopped for anyone under 60 years old (June $11^{\text {th }}$. Therefore, the AstraZeneca affair works as an exemplary case to test either the acceleration, the sensationalisation and the fragmentation of public debate: the enormous attention paid to the campaign both by mass media and citizens, the controversy created around the adverse effects of the vaccine, and the political choices that dictated the timing and modalities of AstraZeneca administration are all elements able to create ideal conditions to intercept the emergence or absence of the aforementioned information disorder phenomena in the Italian hybrid media ecosystem.

\section{A map of the debate based on its digital traces}

To map the online debate around AstraZeneca we decided to collect more than 750.000 tweets and 30.000 news articles for a period of six months ( $1^{\text {st }}$ January $2021-30^{\text {th }}$ June 2021). We have gathered 
tweets using the v2 search API meaning we had access to every public (Italian language in this case) tweet including 'astrazeneca' or 'astra zeneca' in its text. We decided to capture Tweets as opposed to Facebook posts because the social network had grown considerably during the initial phase of the pandemic $\left(+34 \%\right.$ in $\left.2020^{1}\right)$, mainly due to the influx of new users seeking an online arena where to discuss covid-related news. In parallel we used Mediacloud to gather news by using the keyword: 'astrazeneca'. It should be said that Mediacloud contains every piece of news published by nonpaywalled digital sources, meaning the 'online' version of newspapers, while in other contexts this might work as a hindrance, however, it was particularly aligned with our focus.

In order to detect polarization, we have employed a pure retweet network (i.e excluding mentions and comments) as a measurement for ideological affinity, assuming that RT means, most of the times, an endorsement of the original tweet (Metaxas et al 2015). To get a rough measurement of 'ideological' communities we have employed a modularity maximizing community detection tool (Blondel et al. 2008). Finally, we have further triangulated our results with an in depth reading of the top 20 tweets for each community.

\section{Tracking the pace}

A first open research question that guided our analysis concerns the pace of AstraZeneca debate. In order to answer this question, we decided to adopt a traditional strategy: by using temporal information about news and tweets we depicted general trends, intercepting either the pace and the possible accelerations in the production of information and online discussion.

Figure 1: news vs. tweets $^{2}$

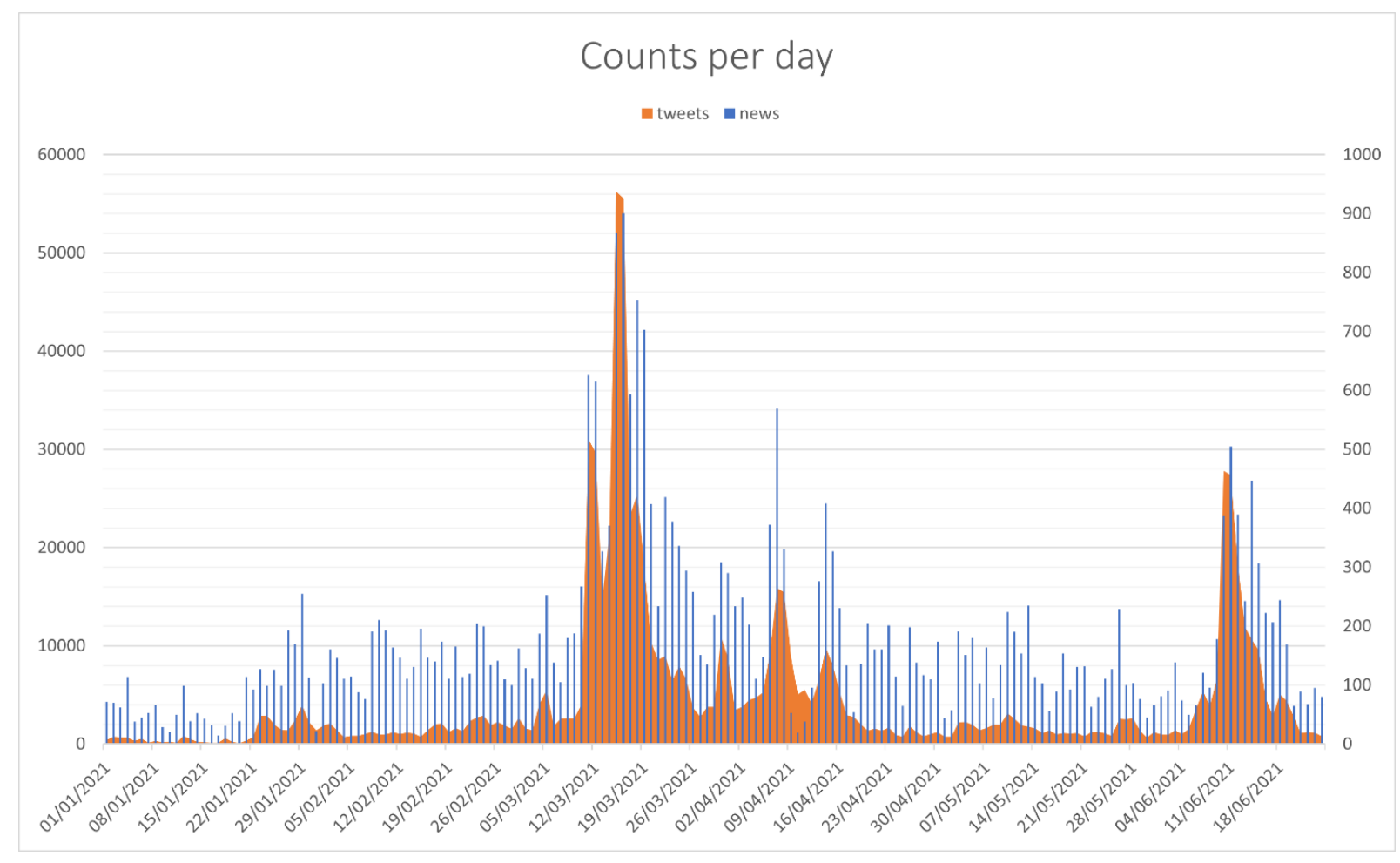

\footnotetext{
${ }^{1}$ https://news.sky.com/story/coronavirus-lockdowns-drive-record-growth-in-twitter-usage-12034770

2 By converting tweets and news counts values to rank order, time series has been normalized on a common scale. The number of tweets is shown on the left and the number of news on the right of the vertical axis.
} 
As shown in Figure 1 the debate on AstraZeneca shows a flat progression all over the 6 months considered, with only three condensed spikes over time: namely from $10 / 03$ to $19 / 03$, from $06 / 04$ to 08/04, and from 10/06 to 15/06. By comparing the historical series of Twitter and MediaCloud it is also possible to clearly distinguish a different pattern of attention paid to vaccine related issues: if the news about AstraZeneca held a consistent level of daily coverage ${ }^{3}$ the average of tweets per day is very reduced ${ }^{4}$. Nevertheless, Twitter discussion presents a sudden and more powerful growth during the peaks of March and June ${ }^{5}$.

\section{Looking for Junk News bubbles}

A second research object concerns the sensationalisation of the issues surrounding the vaccine. To intercept this phenomenon, we decided to circumscribe a sub-sample of the MediaCloud dataset using some specific keywords (i.e., mort*), this way we detected all the news that report in their headlines a story about Astrazeneca suspect deaths. This choice is based on the awareness that the issuance of news related to deaths or serious accidents have become a habitual practice both in tabloid and mainstream journalism, and for this reason news related to AstraZeneca suspect deaths can be seen as a specific sensationalist theme.

Figure 2: news about AstraZeneca vs. news about suspect deaths ${ }^{6}$

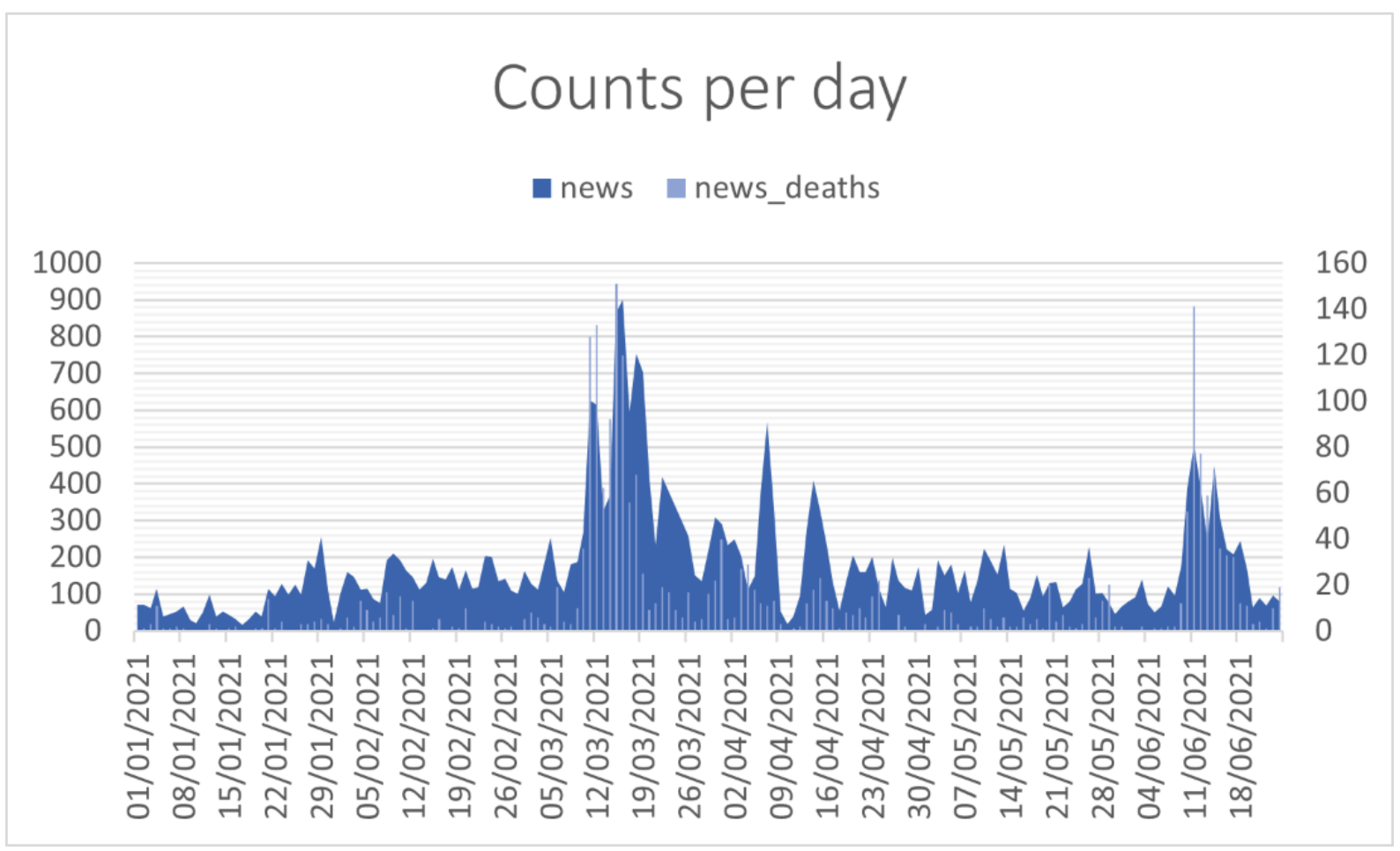

\footnotetext{
${ }^{3}$ On average about 150 news per day are emitted, i.e., approximately $2.5 \%$ of the national sample of news collected by MediaCloud in the chosen period, with a maximum coverage of $10 \%$ during the March peak (see Supplementary Materials Table1).

${ }^{4}$ On average about 2500 tweets per day are posted.

${ }^{5}$ To define a peak of production in the debate, we decided to use a threshold of more than 300 items per day for what concerns news items and a threshold of more than 10.000 posts per day for what regards Twitter.

${ }^{6}$ By converting values to rank order, time series has been normalized on a common scale. The number of news about AstraZeneca is shown on the left and the number of news about suspect deaths on the right of vertical axis.
} 
As shown in Figure 2 for press coverage, during March and June peaks news stories about suspect deaths take a central stage appearing respectively in $15 \%$ and $20 \%$ of total headlines. On the contrary, the central peak of April seems to be generated by a long tail of the controversy regarding the age range in which to administer the vaccine after the stop and go from the $16^{\text {th }}$ to the $19^{\text {th }}$ of March. Indeed, between April $6^{\text {th }}$ and $8^{\text {th }}$, it was firstly issued a rumour on the possible stop of AstraZeneca to people under 60 and then reported the official EMA communication about the correlation between rare thrombosis and AstraZeneca. However, by taking into account the trends shown before in Figure 1 , it is possible to notice that the reaction on Twitter's is not close to the huge volume of tweets emitted in March and June peaks.

Figure 3: news about suspect deaths vs. tweets ${ }^{7}$

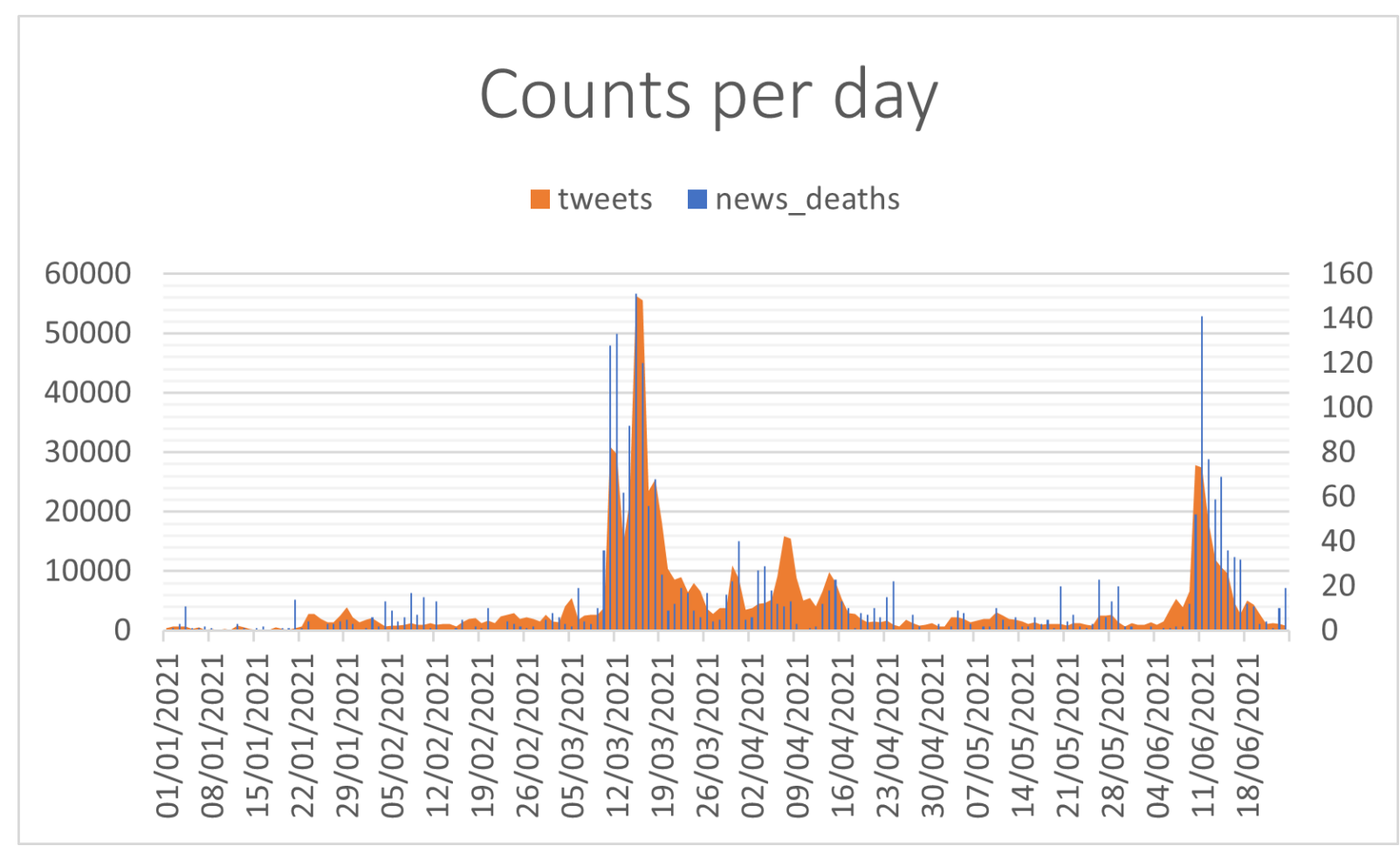

By comparing the trends of Twitter and of the news that report in their headlines a reference to AstraZeneca suspect deaths, Figure 3 shows more clearly that Twitter attention toward AstraZeneca issues is higher and more concentrated on periods when journalistic outlets reported the stories of suspect deaths. As already evidenced in Figure 1, the central peak related to EMA announcement is indeed the most contained both temporally (it lasted only 2 days compared to the 9 days of March peaks and to the 7 day of June peaks) and in terms of volume (about 50.000 tweets versus 200.000 in March or 100.000 in June).

\section{Assessing the reaction of connected publics}

A last area we would like to investigate is the potential fragmentation of public debate. Relying on the connected publics' reaction as a proxy for the fragmentation of online debate, we decided to build

\footnotetext{
${ }^{7}$ By converting values to rank order, time series has been normalized on a common scale. The number of tweets is shown on the left and the number of news about suspect deaths on the right of the vertical axis.
} 
the retweets networks that occurred during the two largest peaks in Twitter, namely the ones of March and June. Firstly, clusters of users within the networks were identified using Louvain algorithm (Blondel et al. 2008) ${ }^{8}$. Then, by calculating the E-I index for each cluster, it was possible to understand which groups of actors were central to the discussion and which groups were instead closed within themselves (Krackhardt and Stern 1988) ${ }^{9}$. Finally, still for each group, the 20 most shared tweets were selected and qualitatively analysed ${ }^{10}$ in order to reconstruct their internal discourse and, consequently, the more general discussion about AstraZeneca during March and June peaks ${ }^{11}$.

First, by looking at the forced-directed network visualization in Figure 4, it is possible to notice how during the first peak of March the discussion on Twitter is strongly polarised, thus being divided into two clearly distinguishable communities. The first one is located on the left of the network visualization and is formed by a single large green cluster. This community is composed of both a few newspapers and politicians belonging to the Italian right-wing parties as well as openly no-vax accounts. The second community, on the right, is instead composed of a multiplicity of clusters, as well as a multiplicity of different types of users. Of these accounts, the most retweeted are major Italian newspapers and television channels, the official accounts of the Police and of the Italian Drug Agency, and a wide variety of opinion leaders consisting of physicians, scientists, and journalists.

Secondly, the E-I index varies substantially for the clusters located at the extremes of the network and for those in the centre. Indeed, the only cluster that has a positive E-I $(+0.65)$ is the central red one made up of mainstream media accounts. For the green cluster instead the E-I is -0.58 , while the remaining clusters of different colours on the right of the visualization have an E-I that range between -0.23 and -0.57 . Therefore, the only positive value assumed by the E-I index shows that the group of users from which the conversation generated are the mainstream media accounts: indeed, to get such a high E-I score, their reports of suspect deaths should have been retweeted by users belonging to all the other clusters identified. As far as these remaining clusters are concerned, by looking at their EI scores it is possible to say that they are mostly inward facing, although this is truer for the green one on the left than for the multi-colours' clusters on the right side of the network.

Digging deeper into more details, the discourses that can be traced through the analysis of the most shared tweets in the different clusters confirm a polarisation not only at network level but also with regard to content. Within the green community on the left of the network, it is possible to trace two different discursive matrices. The first and most evident is what we could define as the no-vax narrative, which is defined by an open accusation against the powers that be (Italy, Europe, or drug firms) who, aware of the damage, want people to be vaccinated anyway. A second narrative within this community is carried by right-wing politicians and is based on an invective against the Italian government, guilty of having made a mistake in the choice of vaccine administration to the point of causing deaths. With regard to the multi-coloured community on the right of the network, as already found for the network metrics, the discourse is more jagged. First of all, there is a discourse led by mainstream media accounts, which after reporting the suspect deaths continue to update the storyline with new events. Another discourse is very similar and concerns tweets related to the official

\footnotetext{
${ }^{8}$ The algorithm maximises the modularity function, which optimizes the density of internal arcs for clusters of users. This way it is possible to carry out a fast recognition of different communities present in a given network.

${ }^{9}$ This parameter measures the portion of internal arcs, i.e., directed from one member to another of the same community, compared to the number of external arcs, i.e., directed from a member of a community to an external member. From this measure, it is possible to convert the number of internal and external arcs into a normalized index, within a spectrum of values between -1 and +1 the E-I index becomes a reliable measure of a node's tendency to connect to nodes within its community.

${ }^{10}$ See Supplementary Materials Table2 for the lists of tweets.

${ }^{11}$ The choice of selecting only the most shared tweets to reconstruct the discussion depends on the so-called law of 1-9-90 (Bracciale et al. 2018), which is also reflected in the Gini index (0.79) of retweets calculated over the entire selected period (1st January 2021 - 30th June 2021).
} 
announcements of the stop-and-go of AstraZeneca administration that are posted by institutional accounts such as the Health Ministry, the Italian Drug Agency, or the Police. Finally, the last and most prevalent discourse in terms of significance relates to the ways in which sensationalist news are debunked by a variety of actors. On the one hand, journalists, physicians, and scientists carry out a rationalising discourse that shows how the risk of death linked to AstraZeneca is actually the same as that of many other common drugs, or even lower compared to others everyday life situations. On the other hand, the same debunking strategy is carried out in a more ironic way by little known users who, however, thanks to their sarcastic tweets, manage to spread their message very widely.

\section{Figure 4: $10^{\text {th }}-19^{\text {th }}$ March retweets network visualization ${ }^{12}$}

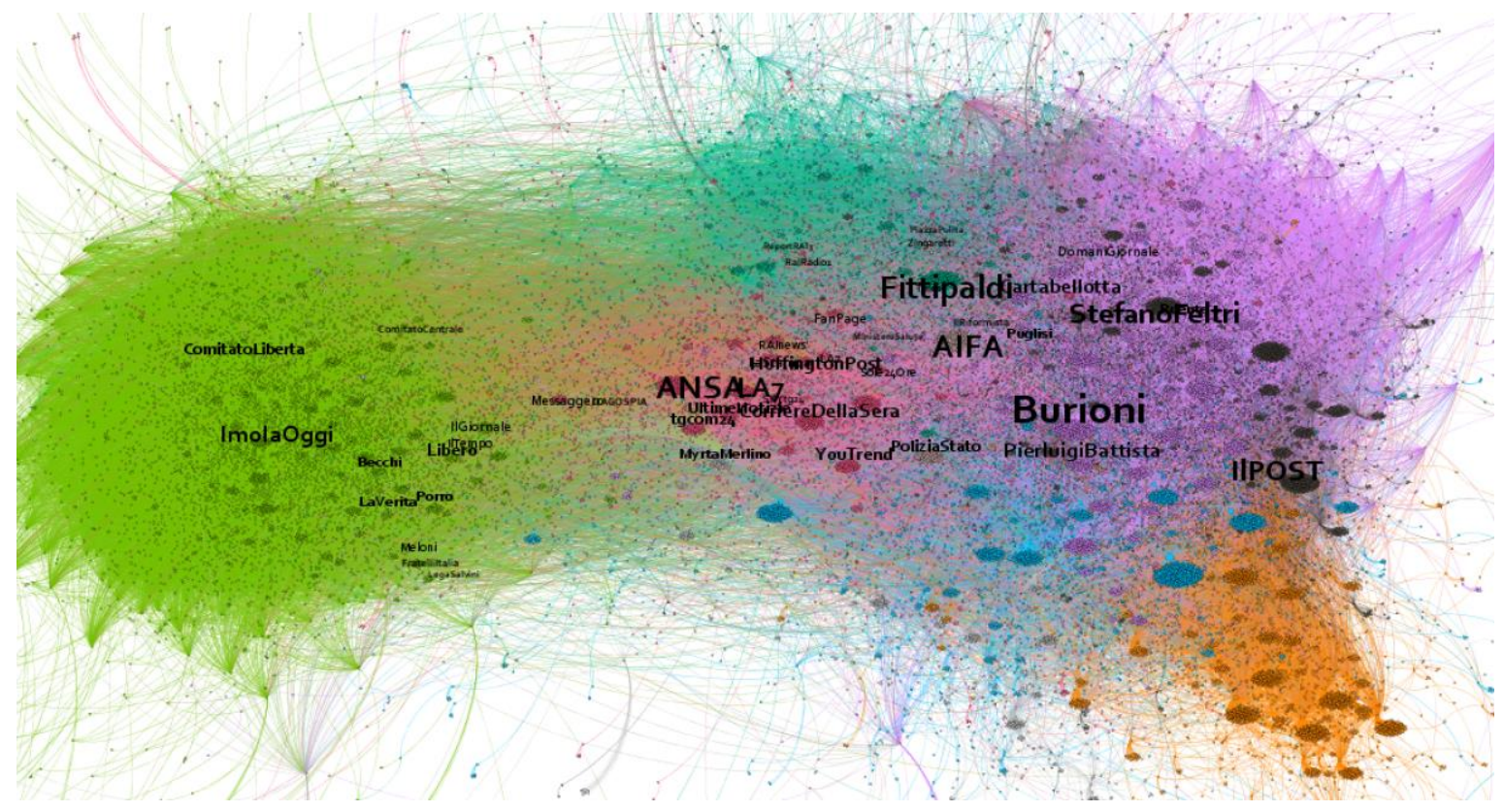

The discussion peak of June presents characteristics that are very similar to the March one, but it accentuates several fragmentation features. First, since the space and distance between clusters in the visualisation of networks by force-directed algorithms indicates how connected they are (Venturini et al. 2021), by looking at Figure 5 it is possible to note how the larger communities displayed by Force Atlas 2 (space) reflect more closely the various clusters identified by Louvain's algorithm (colours). In detail, the green cluster is led by opinion leaders and physicians famous for their debunkers' approach; the blue cluster is centred around some newspapers and journalists close to the M5S; the grey cluster in the middle is instead composed of a multitude of mainstream media accounts; the pink cluster is made up of politicians, journalists and newspapers that are linked to right-wing parties; finally, the purple cluster sees famous no-vax exponents and online hoax sites as its main exponents.

A second feature that is even more pronounced than the first peak is related to the E-I index that measures the openness or closure of the clusters identified by the Louvain algorithm. The grey cluster in the middle, which is composed only by mainstream media, has an E-I index of 0.76 and is therefore

\footnotetext{
${ }^{12}$ Each node represents a user, while an arc between two nodes, whose value is unitary, indicates a retweet of a specific tweet by the user to whom the arc is directed. In order to visualise and analyse the data we used Gephi (Bastian et al. 2009), an opensource software for the analysis and treatment of social networks. The image reported was obtained using the 'Force Atlas 2' visualisation algorithm (Jacomy et al. 2014). The size of the labels is proportional to the number of retweets received. The colours of the arcs represent membership in one of the clusters identified using Louvain's algorithm (Blondel et al. 2008).
} 
even more crucial for the generation of Twitter discussion. As for the other clusters, the E-I index shows a marked negativity and ranges from -0.59 to -0.77 . These scores seem to indicate that, although most users start a discussion from the same news reported by mainstream media accounts, they barely retweet contents from other clusters.

A more partisan dynamic is also confirmed by the analysis of the most shared tweets within each cluster. As with the first peak, during the spikes of June mainstream media accounts reported a continuous flow of news and updates on AstraZeneca-related deaths. However, the prevailing discourses in other communities appear more radicalised. Within the blue cluster, the most shared tweets generate from a statement by the journalist Marco Travaglio who point out that if the mistake of the open nights' events happened when Conte was prime minister, the press and the parties would never have forgiven him. The green cluster, always composed of opinion leaders who seek to debunk an excessive exaggeration of the link between AstraZeneca and thrombosis' deaths, is driven by a rationalizing discourse that also try to explain the concusses of some deaths due to the AstraZeneca vaccine. Finally, the purple and pink clusters see a greater separation of no-vax narratives and rightwing parties: the former continue to accuse the system of deliberately wanting to kill people, while politicians and journalists call for the resignation of the Italian health minister and for the individual freedom of not taking any vaccine.

Figure 5: $10^{\text {th }}-17^{\text {th }}$ June retweets network visualization ${ }^{13}$

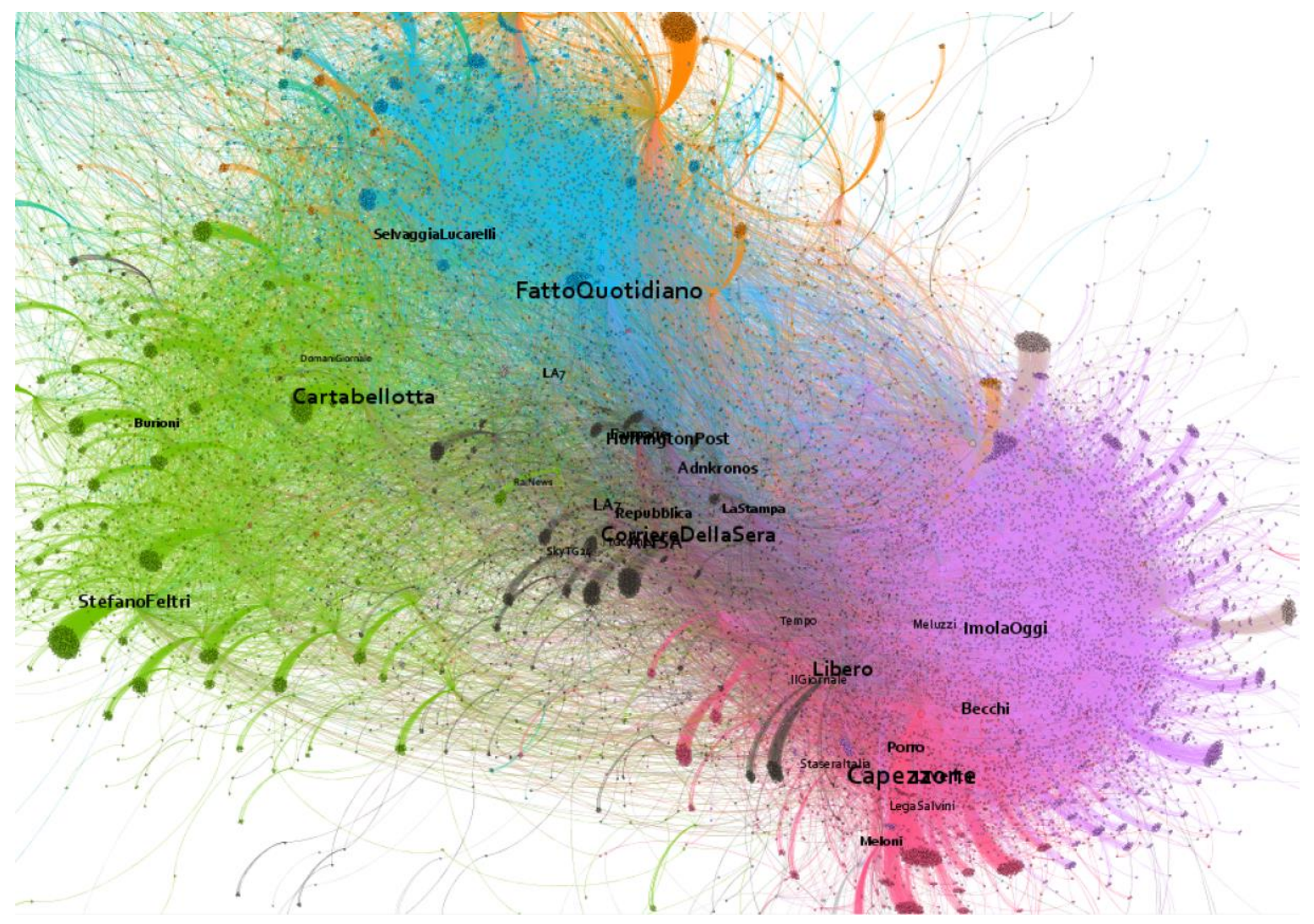

\footnotetext{
${ }^{13}$ Each node represents a user, while an arc between two nodes, whose value is unitary, indicates a retweet of a specific tweet by the user to whom the arc is directed. In order to visualise and analyse the data we used Gephi (Bastian et al. 2009), an opensource software for the analysis and treatment of social networks. The image reported was obtained using the 'Force Atlas 2' visualisation algorithm (Jacomy et al. 2014). The size of the labels is proportional to the number of retweets received. The colours of the arcs represent membership in one of the clusters identified using Louvain's algorithm (Blondel et al. 2008).
} 


\section{Acceleration, Sensationalisation, and Fragmentation: three intertwined information disorders}

At this point, the results obtained allow us to precisely outline the emergence of information disorder in the Italian debate on AstraZeneca, and to understand how the intertwining between them can help us to dig deeper into the logic of the hybrid media ecosystem in which they take shape.

A first important finding concerns the trends of news coverage and of Twitter discussion: indeed, legacy, and social media temporal dynamics are tightly connected and seem to influence one another. Although news coverage is constant on AstraZeneca, it is only when it grows disproportionately that the discussion on Twitter takes off. Such a dynamic of disinterest of the connected publics towards the daily news reporting appears to explain as well the sensationalisation phenomenon: it is indeed when news about suspect deaths are mostly reported in the headlines that the bursts and accelerations previously described get going and the connected publics aggregate around legacy media agenda. From this point of view, even though these two different phenomena of information disorder have often been detected and analysed, the case study of AstraZeneca highlights how one possible explanation for their co-presence is caused properly by the junk news attention regime (Venturini 2019). Indeed, by accelerating the turnover of online content, Web 2.0 reduces the time for discussion of each topic (Castaldo et al. 2020). This faster scheme causes a shift in the attention of the connected public towards more extreme content, such as sensationalist news (Chadwick et al. 2018). The example of AstraZeneca shows how this kind of dynamic emerges also in the Italian hybrid media ecosystem: by reducing the space for debate to sensationalism contents only, such a conformation can led to a more overbearing of confirmation biases and ideological positions that are already embedded in social media (Del Vicario et al. 2017).

A second finding precisely concerns this online discussion mechanism. The analysis of Twitter shows that during the two peaks of attention, the connected publics split into opposite factions. This immediate polarisation is made even more evident by the discourses present in the different clusters: the rhetoric used is in fact simple and is based on ideological divisions that were already evident in the general debate on the covid-19 and preceded the AstraZeneca affair (Caliandro et al. 2020). Nevertheless, as the attention bursts caused by news about the suspect deaths continue, the ideological polarisation seems to become even more pronounced. A comparison of the March and June networks shows that from the initial two hubs the fragmentation in Twitter expands to four poles. This is also confirmed by the evolution of online discourses, which become increasingly distinct and exclusive as time goes by.

As with the phenomenon of acceleration and sensationalism, also fragmentation has predominantly negative effects on the quality of public debate. Indeed, to have a prolonged and fruitful consultation between different factions, a form of continuous mediation is required. This mediative role used to be delegated to journalists and mainstream media themselves. However, the AstraZeneca case highlights how traditional journalism struggles to cover such a controversial issue and at the same time entering the new business model of the platform society (Van Dijck et al. 2018). In this sense the three information disorder studied are at the very basis of the decline of public debate quality: on one hand the hit economy leads to a constant acceleration of debate, forcing the production of increasingly catchy content; on the other part, by feeding confirmation bias, sensationalist news leads to an increasing polarisation and closure of social media users. This continuous and repeated cycle thus leads to an atrophied and sluggish public debate. 


\section{Conclusion}

Within media studies, and in particular for the branch that uses digital methods as its main research tool, the infodemic has been at the centre of academic disputes since the dawn of the covid-19 outbreak. Although the overabundance of information related to the virus may be a significant object of study in itself, our intention has been to turn this situation into a unique opportunity to better understand the logic and fallacies of hybrid media ecosystems, in the conviction that this category defines and will continue to define the digital society in a more fundamental way. Indeed, the case we chose could be considered as a possible litmus test of the information disorder that takes place in the Italian media system. In detail, through the analysis carried out it was possible to check if and how the phenomena of acceleration, sensationalisation and fragmentation of public debate occurred. As far as the Italian debate on the AstraZeneca vaccine is concerned, our results suggest an affirmative answer to this question and indicate that all the three phenomena are simultaneously present and intertwined. Despite this peculiar event cannot be generalised, we believe that the AstraZeneca affair shows how the listed information disorder phenomena occur and interact. The lack of attention of connected publics towards traditional coverage may in fact explain journalism's search for sensationalistic scoop. However, the emission of such news correlates with a spiked but limited debate, which pushes towards heuristic shortcuts in information processing. Such biases can be traced back properly in Twitter discussion, that is characterized by a strong and growing fragmentation over time and by a consistent hierarchy of the conversation around a few influential users. Taken together, these information disorder phenomena can be detrimental to the formation of a public arena capable of sustaining fruitful debate on highly controversial social issues such as covid-19 vaccines. Slowness to assimilate information and not bursts of attention, impartiality to detail reality and not sensationalism, and sharing a common space of conversation in opposition to online tribalism may be the only ways to rebuild a healthy and robust democratic space.

\section{References}

Bastian, M., Heymann, S., \& Jacomy, M. (2009). Gephi: an open-source software for exploring and manipulating networks. In Third international AAAI conference on weblogs and social media.

Blondel, V. D., Guillaume, J. L., Lambiotte, R., \& Lefebvre, E. (2008). Fast unfolding of communities in large networks. Journal of statistical mechanics: theory and experiment, 2008(10), P10008.

Bracciale, R., Martella, A., \& Visentin, C. (2018). From Super-Participants to Super-Echoed. Participation in the 2018 Italian Electoral Twittersphere. PARTECIPAZIONE E CONFLITTO, 11(2), 361-393.

Boccia Artieri, G. (2020). A Voce Alta. Pandemic politics: un nuovo campo discorsivo per la ricerca sulla politica. Comunicazione politica, 21(3), 443-449.

Boccia Artieri, G., García-Bilbao, P. A., \& La Rocca, G. (2021). Rethinking affective polarization and sharing of emotions in digital platform ecosystems. Theories and research practices. International Review of Sociology/Revue Internationale de Sociologie, 31(2), 223-230.

Caliandro, A., Anselmi, G., \& Sturiale, V. (2020). Fake news, Covid-19 e Infodemia: un esempio di ricerca sociale in real-time su Twitter. Mediascapes journal, (15), 174-188. 
Castaldo, M., Venturini, T., Frasca, P., \& Gargiulo, F. (2020). Junk news bubbles modelling the rise and fall of attention in online arenas. New Media \& Society, 1461444820978640.

Chadwick, A., Vaccari, C., \& O'Loughlin, B. (2018). Do tabloids poison the well of social media? Explaining democratically dysfunctional news sharing. New Media \& Society, 20(11), 4255-4274.

Cinelli, M., Quattrociocchi, W., Galeazzi, A., Valensise, C. M., Brugnoli, E., Schmidt, A. L., ... \& Scala, A. (2020). The COVID-19 social media infodemic. Scientific Reports, 10(1), 1-10.

Del Vicario, M., Scala, A., Caldarelli, G., Stanley, H. E., \& Quattrociocchi, W. (2017). Modeling confirmation bias and polarization. Scientific reports, 7(1), 1-9.

Eysenbach, G. (2002). Infodemiology: The epidemiology of (mis) information. The American journal of medicine, 113(9), 763-765.

Simon, F. M., \& Camargo, C. Q. (2021). Autopsy of a metaphor: The origins, use and blind spots of the 'infodemic'. new media \& society, 14614448211031908.

Gallotti, R., Valle, F., Castaldo, N., Sacco, P., \& De Domenico, M. (2020). Assessing the risks of 'infodemics' in response to COVID-19 epidemics. Nature Human Behaviour, 4(12), 1285-1293.

Graham, R. (2017). Google and advertising: digital capitalism in the context of PostFordism, the reification of language, and the rise of fake news. Palgrave Communications, 3(1), 1-19.

Gray, J., Bounegru, L., \& Venturini, T. (2020). 'Fake news' as infrastructural uncanny. New media \& society, 22(2), 317-341.

Gruzd, A., De Domenico, M., Sacco, P. L., \& Briand, S. (2021). Studying the COVID-19 infodemic at scale. Big Data \& Society, 8(1), 20539517211021115

Iannelli, L., Splendore, S., Valeriani, A., \& Marino, G. (2020). Studiare la polarizzazione politica nello "shockdown mediale". Mediascapes journal, (15), 189-202.

Metaxas, P., Mustafaraj, E., Wong, K., Zeng, L., O'Keefe, M., \& Finn, S. (2015, April). What do retweets indicate? Results from user survey and meta-review of research. In Proceedings of the International AAAI Conference on Web and Social Media (Vol. 9, No. 1).

Jacomy, M., Venturini, T., Heymann, S., \& Bastian, M. (2014). ForceAtlas2, a continuous graph layout algorithm for handy network visualization designed for the Gephi software. PloS one, 9(6), e98679.

Krackhardt, D., \& Stern, R. N. (1988). Informal networks and organizational crises: An experimental simulation. Social psychology quarterly, 123-140.

Marwick, A., \& Lewis, R. (2017). Media manipulation and disinformation online. Data \& Society.

Righetti, N., Rossi, L., \& Marino, G. (2021). Into the belly of the beast: the research on social media and covid-19 misinformation in 2020. AoIR Selected Papers of Internet Research.

Rojecki, A., \& Meraz, S. (2016). Rumors and factitious informational blends: The role of the web in speculative politics. New Media \& Society, 18(1), 25-43.

Saracino, B. (2020). Comunicare la scienza al tempo del Coronavirus: tra domanda e offerta di informazione. Associazione Italiana di Sociologia, (16), 99-119 
Van Dijck, J., Poell, T., \& De Waal, M. (2018). The platform society: Public values in a connective world. Oxford University Press.

Venturini, T. (2019). From fake to junk news: The data politics of online virality. In Data Politics (pp. 123-144). Routledge.

Venturini, T., Jacomy, M., \& Jensen, P. (2021). What do we see when we look at networks: Visual network analysis, relational ambiguity, and force-directed layouts. Big Data \& Society, 8(1), 20539517211018488.

Wardle, C. (2017). Fake news. It's complicated. First Draft, 16, 1-11.

Zarocostas, J. (2020). How to fight an infodemic. The lancet, 395(10225), 676. 